\title{
COMPUTATIONAL APPROACHES TO IDENTIFY A DERIVATIVE OF GALARDIN AS AN INHIBITOR OF MYCOBACTERIAL PEPTIDE DEFORMYLASE
}

\author{
ASIF HASSAN SYED $^{1^{*}}$, IFTEKHAR ASLAM TAYUBI ${ }^{2}$ \\ ${ }^{1 *}$ Department of Computer Science, Faculty of Computing and Information Technology, Rabigh, King \\ Abdulaziz University, Jeddah, Saudi Arabia \\ ${ }^{2}$ Department of Computer Science, Faculty of Computing and Information Technology, Rabigh, King \\ Abdulaziz University, Jeddah, Saudi Arabia \\ Email: shassan1@kau.edu.sa
}

\begin{abstract}
Revised February 2017
ABSTRACT. The metalloenzyme peptide deformylase (PDF) catalyzes the elimination of the $\mathrm{N}$-formyl group from $\mathrm{N}$-terminal methionine upon translation, which is crucial for protein synthesis, growth, and survival of bacteria. In this context, we aim to identify potent derivatives of the known mycobacterial PDF (mPDF) inhibitors having better pharmacological properties than their parent compounds. Initially, BB-83698, Galardin, and LBK-611 known mPDF inhibitors were selected based on their binding affinity for mPDF using iGEMDOCK. Analogs of these three inhibitors were prepared. Further, the analogs were screened based on their oral bioavailability, pharmacokinetics properties, drug likeliness and binding energy. The post-screening analysis reveals that the analog, (2R)-N'-hydroxy-N-[(2S)-3-(5H-indol-3-yl)-1-oxopropan-2-yl]-2-(2-methylpropyl) butanediamide (CID5288446) of galardin interacts with residues GLN56, LEU107, HIS148, GLU149, and HIS152 near the vicinity of the active site $\left(\mathrm{H}^{132} \mathrm{EXXH}^{136}\right)$ of mPDF protein with higher affinity as compared to its parent compound galardin. The prediction tool based upon structure-activity relationship reveals that the analog CID5288446 showed similar metalloproteinase activity with lesser toxic effects when compared to its parent compound galardin.

Keywords: Mycobacterial Peptide Deformylase (mPDF); structure-based virtual screening; fitness score; pharmacokinetics properties; drug likeliness; Inhibitors.
\end{abstract}

1. Introduction. Tuberculosis (TB) is an infectious disease that has afflicted humans since the Neolithic period. Mycobacterium tuberculosis (MTB) is a pathogenic bacteria belonging to the genus Mycobacterium and is the principal agent responsible for maximum cases of Tuberculosis (TB) [1]. The advent of multi-drug resistant (MDR) strains of $M T B$ provides a real challenge to successfully treat tuberculosis infection. There were around 190000 deaths from MDR-TB and roughly 480000 new cases of MDR-TB worldwide in the year 2014 [2]. In order to address this problem, the identification of novel antibiotic against drug-resistant strains of $M T B$ is crucial to ensure the future success of therapeutics against MTB. Recently, PDF a metalloenzyme crucial for deformylation and maturation of bacterial protein have emerged as a potential target to develop novel antimycobacterial agents with a new mode of action $[3,4,5]$. Catalytic removal of the $\mathrm{N}$-formyl group from $\mathrm{N}$-terminal methionine upon translation is essential for the growth and viability of $M T B$ $[6,7]$. Using rationally designed screening protocols many novel classes of inhibitors specific to PDF have been identified namely BB-3497 and actinonin $[8,9]$. Even many of the derivatives of actinonin namely VIC104959 (LBM 415) and BB-83698 have progressed on to phase II and III trials against clinical strains of Haemophilus influenza, Enterococci, Staphylococci and Streptococci [10,11, 12, 13]. Even though quite a few new anti-PDF drugs have reached to clinical trials, but their usage against mycobacterial PDF is difficult 
to predict. Therefore, there is an urgency of screening novel derivatives of potent PDF inhibitors with improved pharmacokinetic and binding properties against mPDF protein. In this context, we were able to screen an analog of a well-known mPDF inhibitors and evaluated its` affinity for mPDF. Our in silico study of mPDF inhibitors will certainly add on to the list of the probable lead compound against mPDF protein and make sure that the pipeline of novel lead compounds against mPDF protein continues.

\section{Methods.}

Virtual Screening using iGEMDOCK: The docking tool iGEMDOCK v2.0 was used to perform rapid virtual screenings of the mPDF inhibitors compounds against mPDF protein $[14,15,16]$ using the crystal structure of MTB peptide deformylase [4]. The following four major steps are involved in virtual screening using iGEMDOCK: (1) retrieval of target protein structure; (2) preparing compound library; (2) Preparation of binding site; (3) protein-ligand docking and (4) Docked poses/post-screening analysis.

Retrieval and preparation of energy minimized structure of target protein: The crystal structure of MTB peptide deformylase with PDB ID 3E3U (resolution $1.56 \AA$ ) with an inhibitor was retrieved from Research Collaboratory for Structural Bioinformatics Protein Data Bank (RCSB PDB) (http://www.rcsb.org/pdb/home/ home.do). The ready to dock minimized structure of PDF protein was prepared using the Dock Prep tool of Chimera 1.10.2.

Ligand preparation: The 3D structural files of seven known mPDF inhibitors namely actonin, BB-3497, galardin, LBK-611, N-alkyl urea hydroxamic acids, VRC-3324 and VRC-3325 and their corresponding analogs were downloaded from PubChem database (https://pubchem.ncbi.nlm.nih.gov/compound/). The structures downloaded were in .sdf format. OpenBabel (http://openbabel.org/wiki/Main_Page) software was used to convert the.$s d f$ files to .mol2 format since iGEMDOCK docking tool needs structural files in .mol2 format for executing molecular docking calculations. Ready to use dock prep structures of PDF inhibitors were prepared using Chimera 1.10.

Ligand Binding Site Preparation: The mPDF protein in complex with inhibitor was downloaded in .pdb format from RCSB PDB (PDB ID 3E3U). The binding region of the bounded inhibitor was defined as the binding site for virtual screening. The bounded inhibitor was defined as the center of the binding domain and the size of the binding position was set to a default value of $8 \AA$.

Ligand-protein docking of known PDF inhibitors: Standard docking protocol of iGEMDOCK v2.0 was used to screen inhibitor compounds having a higher affinity for mPDF protein. A population size of 200 with 70 generation and two solutions for each generation was set for molecular docking studies. Top three mPDF inhibitors were selected based on their affinity for the ligand binding domain of the crystal structure of the mPDF protein.

Analog preparation and virtual screening: A library of analogs of top three mPDF inhibitors screened based on their affinity for mPDF protein was retrieved from PubChem, dock prepared using chimera 1.10.2 and screened against the crystal structure of the mPDF protein using the standard docking procedure of iGEMDOCK. The top ten analogs selected based on their affinity for the targeted ligand binding domain of mPDF protein were further checked for their pharmacological and drug-likeliness properties.

Drug-Likeness and Pharmacological Analysis of screened analogs: The physiochemical descriptors of the selected ten ligands were estimated for oral drug availability, drug-likeness and pharmacokinetics properties of the screened ligands using SwissADME (http://www.swissadme.ch/index.php). On the basis of binding affinity, drug-likeliness, and ADME properties, eight ligands were selected for very accurate docking studies.

Molecular docking and post-dock interaction studies of screened ligand and its parental compound: The screened drug-like lead analog molecule and its parent compound were subjected to the very slow docking (accurate docking) procedure of iGEMDOCK. Once molecular docking was completed, protein-ligand interaction profile consisting of Van der Waal's (V), hydrogen-bonding $(\mathrm{H})$ and electrostatic (E) was generated. Based on these profiles the compounds are compared using the energy-based scoring function iGEMDOCK [16]. 
Visualization of the interacting residues: The 2-D representation of the interacting residues of the docked complexes was generated by LIGPLOT PLUS program [17]. The pictorial representation was helpful in determining the interacting functional groups of the novel drug-like lead molecule with the target protein.

Prediction of biological activity and toxicity of the screened drug-like molecule and its parental compound: PASS (Prediction of Activity Spectra for Substances) software based on the structure-activity relationship $[18,19]$ was used to predict the biological activity and potential toxic effects of both the screened drug-like ligand and its parental molecule.

\section{Results and Dicussion}

Virtual screening analysis: Based upon their total binding energy (affinity) for mPDF protein top three PDF inhibitors namely BB-83698 (-143.679 kcal/mol), Galardin (-133.186 kcal/mol) and LBK-611 (128.687 $\mathrm{kcal} / \mathrm{mol}$ ) were selected using standard screening protocol of iGEMDOCK and tabulated in Table 1. Further, ten best derivatives (analogs) derived from parent inhibitor molecules (BB-83698, Galardin, and LBK-611) were chosen based on their affinity (binding energy) for mPDF protein and are listed in Table 2. It can be observed that derivatives CID 20752846 and CID 5288446 of BB-83698 and Galardin, respectively showed a better binding affinity for mPDF protein when compared to other derivatives of same or other parent inhibitor molecules.

Table 1: Binding Energies for the known mPDF inhibitors against the crystal structure of mPDF using the standard docking protocol of iGEMDOCK

\begin{tabular}{clcccc}
\hline Sl. No. & \multicolumn{1}{c}{ mPDF inhibitors } & Total Energy & VDW & HBond & Elec \\
\hline 1 & BB-83698 & -139.679 & -123.232 & -16.447 & 0 \\
2 & Galardin & -127.186 & -107.735 & -19.4516 & 0 \\
3 & LBK611 & -125.687 & -94.0976 & -33.5896 & 0 \\
4 & Actinonin & -124.614 & -92.9471 & -31.6666 & 0 \\
5 & LBM 415 & -112.225 & -93.0621 & -19.1625 & 0 \\
6 & VRC-3324 & -111.277 & -96.723 & -14.5543 & 0 \\
7 & VRC-3375 & -119.256 & -90.4762 & -28.7797 & 0 \\
8 & N-alkyl urea hydroxamic acids & -110.285 & -90.108 & -20.177 & 0 \\
9 & BB-3497 & -102.737 & -95.9264 & -6.81028 & 0 \\
\hline
\end{tabular}

Table 2: Binding Energies for the ten best analogs of mPDF inhibitors docked against the crystal structure of mPDF protein

\begin{tabular}{ccccccc}
\hline Sl. No. & $\begin{array}{c}\text { Analog of } \\
\text { mPDF inhibitors }\end{array}$ & $\begin{array}{c}\text { Parent connectivity } \\
\text { (mPDF inhibitors) }\end{array}$ & Total Energy & VDW & HBond & Elec \\
\hline 1 & CID 20752846 & BB-83698 & -140.526 & -114.41 & -26.1165 & 0 \\
2 & CID 5288446 & Galardin & -136.663 & -102.282 & -34.3812 & 0 \\
3 & CID 20752877 & BB-83698 & -135.494 & -112.384 & -23.1102 & 0 \\
4 & CID 23520197 & LBK-611 & -132.242 & -103.057 & -29.1846 & 0 \\
5 & CID 74332344 & LBK-611 & -129.252 & -105.001 & -24.2513 & 0 \\
6 & CID 10346022 & BB-83698 & -127.805 & -119.347 & -8.45779 & 0 \\
7 & CID 510181 & BB-83698 & -127.12 & -101.409 & -25.7106 & 0 \\
8 & CID 58763319 & LBK-611 & -122.284 & -103.303 & -18.9816 & 0 \\
9 & CID 86706411 & LBK-611 & -121.59 & -88.4755 & -33.1149 & 0 \\
10 & CID 74332343 & LBK-611 & -121.21 & -97.4957 & -23.7147 & 0 \\
\hline
\end{tabular}

Oral bioavailability and drug-likeness studies: The physiochemical properties showing the oral bioavailability and drug-likeness of the ten best-screened ligands were evaluated using SwissADME server and are tabulated in Table 3. 
Table 3: The physiochemical properties depicting the oral bioavailability and drug-likeliness of the ten best-fitted analogs of mPDF inhibitors

\begin{tabular}{|c|c|c|c|c|c|c|c|c|c|}
\hline $\begin{array}{l}\text { Sl. } \\
\text { No }\end{array}$ & Molecule & $\overline{M W}$ & $\begin{array}{l}\text { Fraction } \\
\text { Csp3 }\end{array}$ & $\begin{array}{l}\text { Rotatable } \\
\text { bonds }\end{array}$ & $T P S A$ & $X L O G P 3$ & $\begin{array}{l}E S O L \\
\log S\end{array}$ & $\begin{array}{l}\text { Lipinski } \\
\text { violations }\end{array}$ & Drug-Likeness \\
\hline 1 & $\begin{array}{l}\text { CID } \\
23520197\end{array}$ & 395.4 & 0.53 & 11 & 119.0 & 1.4 & -2.6 & None & Yes \\
\hline 2 & $\begin{array}{l}\text { CID } \\
74332344\end{array}$ & 363.4 & 0.59 & 11 & 115.7 & 0.37 & -1.8 & None & Yes \\
\hline 3 & $\begin{array}{l}\text { CID } \\
5288446\end{array}$ & 388.5 & 0.5 & 8 & 119.9 & 0.24 & -1.6 & None & Yes \\
\hline 4 & $\begin{array}{l}\text { CID } \\
58763319\end{array}$ & 376.5 & 0.58 & 11 & 102.8 & 1.74 & -2.7 & None & Yes \\
\hline 5 & $\begin{array}{l}\text { CID } \\
86706411\end{array}$ & 387.4 & 0.53 & 10 & 124.0 & 0.68 & -2.2 & None & Yes \\
\hline 6 & $\begin{array}{l}\text { CID } \\
74332343\end{array}$ & 363.4 & 0.59 & 11 & 115.7 & 0.31 & -1.7 & None & Yes \\
\hline 7 & $\begin{array}{l}\text { CID } \\
20752846\end{array}$ & 536.71 & 0.58 & 13 & 93.19 & 4.93 & -5.61 & 1 & Yes \\
\hline 8 & $\begin{array}{l}\text { CID } \\
20752877\end{array}$ & 559.7 & 0.66 & 15 & 122.73 & 3.51 & -4.64 & 1 & Yes \\
\hline 9 & $\begin{array}{l}\text { CID } \\
10346022\end{array}$ & 504.62 & 0.65 & 14 & 111.65 & 2.91 & -4 & 1 & Yes \\
\hline 10 & $\begin{array}{l}\text { CID } \\
510181\end{array}$ & 493.66 & 0.75 & 13 & 134.32 & 2.75 & -3.88 & None & Yes \\
\hline
\end{tabular}

Oral bioavailability of the ligands was evaluated based on the threshold value of certain physiochemical descriptors namely Lipophilicity $(-0.7<$ XLOGP3 $<+5.0)$, Size $(150 \mathrm{~g} / \mathrm{mol}<\mathrm{MV}<500 \mathrm{~g} / \mathrm{mol})$, Polarity (20 $\AA^{2}<$ TPSA $\left.<130 \AA^{2}\right)$, Insolubility $(0<\log \mathrm{S}($ ESOL $)<6)$, Instauration $(0.25<$ Fraction Csp $3<1)$ and Flexibility $(0<$ Num. rotatable bonds $<9)$ of the SwissADME server. It can be observed fromTable 3 that all the chemical analog molecules except CID 5288446 show a violation in the number of rotatable bonds $(>10)$. Moreover, chemical analogs CID 20752846, CID 20752877 and CID 10346022 show an additional violation of molecular mass $(>500 \mathrm{~g} / \mathrm{mol})$. While the chemical analog CID 10346022 shows a violation in molecular mass $(>500 \mathrm{~g} / \mathrm{mol})$, a number of rotatable bonds $(>10)$ and polarity (Total Polar Surface Area (TPSA) $>130$ A2). Therefore in this background, we can predict that the chemical analog CID 5288446 which is a derivative of galardin shows better oral bioavailability properties than the other analog molecules. Additionally, druglikeness of the chemical analogs was also evaluated using the Lipinski's rule of five (RO5) [20]. As per RO5, a chemical compound to be orally active in human should follow minimum three criteria of the following: (a) molecular weight $\leq 500$, (b) XLOGP3 $<3.5$, (c) hydrogen bond acceptor $\leq 10$ and (d) hydrogen bond donor $\leq 5$. Therefore in this context, it was observed that the chemical compounds namely CID 74332343, CID 23520197, CID 5288446, CID 58763319, CID 86706411, CID 510181 had zero violation of the RO5. On the contrary molecules CID 20752846, CID 10346022 and CID 20752877 had no more than one violation (Mol. Wt. > 500) of the above criteria. Since all these analog molecules satisfy RO5, therefore, they were classified as virtual drug-like molecules.

Pharmacokinetics assessment of the analogs: The critical pharmacokinetic properties of the analogs relating to its Gastro Intestinal (GI) absorption, Blood Brain Barrier (BBB) permeation, drug metabolism, and permeability glycoprotein activity are highlights in Table 4 . 
Table 4: The pharmacokinetics properties of the top ten best-fitted analogs of mPDF inhibitors

\begin{tabular}{llllllllll}
\hline $\begin{array}{l}\text { Sl. } \\
\text { No }\end{array}$ & Molecule & $\begin{array}{l}\text { GI } \\
\text { absorption }\end{array}$ & $\begin{array}{l}\text { BBB } \\
\text { permeant }\end{array}$ & $\begin{array}{l}\text { CYP1A2 } \\
\text { inhibitor }\end{array}$ & $\begin{array}{l}\text { CYP2C19 } \\
\text { inhibitor }\end{array}$ & $\begin{array}{l}\text { CYP2D6 } \\
\text { inhibitor }\end{array}$ & $\begin{array}{l}\text { CYP3A4 } \\
\text { inhibitor }\end{array}$ & $\begin{array}{l}\text { CYP2C9 } \\
\text { inhibitor }\end{array}$ & $\begin{array}{l}\text { Pgp } \\
\text { substrate }\end{array}$ \\
\hline 1 & $\begin{array}{l}\text { CID } \\
23520197\end{array}$ & High & No & No & No & Yes & Yes & Yes & Yes \\
2 & $\begin{array}{l}\text { CID } \\
74332344\end{array}$ & High & No & No & No & No & No & No & Yes \\
3 & $\begin{array}{l}\text { CID } \\
5288446\end{array}$ & High & No & No & No & No & No & No & No \\
4 & $\begin{array}{l}\text { CID } \\
58763319\end{array}$ & High & No & No & No & No & No & No & Yes \\
5 & $\begin{array}{l}\text { CID } \\
86706411\end{array}$ & High & No & No & No & No & No & No & Yes \\
6 & $\begin{array}{l}\text { CID } \\
74332343\end{array}$ & High & No & No & No & No & No & No & Yes \\
7 & $\begin{array}{l}\text { CID } \\
20752846\end{array}$ & High & No & No & No & Yes & Yes & Yes & Yes \\
8 & $\begin{array}{l}\text { CID } \\
20752877\end{array}$ & High & No & No & No & No & No & Yes & No \\
9 & $\begin{array}{l}\text { CID } \\
10346022\end{array}$ & High & No & No & No & No & No & Yes & Yes \\
10 & $\begin{array}{l}\text { CID } \\
510181\end{array}$ & High & No & No & No & No & No & Yes & Yes \\
\hline
\end{tabular}

From the Table 4, it is observed that all the analogs have high gastrointestinal absorption capability and therefore can be easily translocated to the blood stream. Ideally, the chemical analog compound should target other parts of the human body except for the brain therefore preferably the analogs should not cross the BBB to avoid possible neurological side effects [21]. In the present study, all the screened analogs were predicted to be nonpermeable to $\mathrm{BBB}$, therefore, it avoids the possible psychotropic side effects associated with non-neuroactive drugs permeable to BBB. Metabolic clearance of the drug from the body is an important pharmacokinetic property of any drug molecule. Therefore the roles of cytochromes P450 (CYPs) drug metabolizing enzymes are essential for the metabolism and clearance of the drug from the human system [22]. In this case, the ligands CID 20752846, CID 510181, CID 20752877 and CID 10346022 inhibits the members of cytochrome p450 family of oxidizing enzymes namely CYP2D6, CYP3A4 and CYP2C9 which play an important role in drug metabolism. Since the proper functioning of CYPs enzymes is essential for the metabolic clearance of the drug from the body. Therefore the inhibition of these enzymes by the analog molecules might result in increased bioavailability and the strong possibility of overdosing and eventually toxicity. However, the analogs CID 23520197, CID 74332344, CID 5288446, CID 58763319, CID 86706411 and CID 74332343 are non-inhibitors of drug metabolizing enzymes, therefore, have good pharmacokinetic properties. P-glycoprotein (P-gp) is a transmembrane permeability glycoprotein that functions as a primary active efflux transporter. A number of important drugs are substrates to P-gp and that negatively affect their bioavailability and resistance is induced because of the effluxing nature of the protein. Therefore chemical compound which is non-substrate of P-gp protein is expected to overcome the poor bioavailability and multidrug resistance problems [23]. In this context, the derivative of galardin (CID 5288446) was predicted to be a non-substrate for P-gp protein thus negating the efflux mechanism of P-gp protein. Thereby the drug-like, lead molecule CID 5288446 may overcome the bioavailability and multi-drug resistance issues associated with anti-tuberculosis drugs.

Molecular docking analysis of lead drug-like molecule and its parent compound: Molecular docking of both the parent compound (galardin) and the drug-like lead derivative molecule (CID 5288446) of galardin was performed against the crystal structure of mPDF using the very slow (accurate) docking protocol of iGEMDock2.1. The interaction of galardin and its analogs with mPDF protein was estimated using total 
binding, van der Waals interaction (VDW) and Hydrogen bonding interaction energy. The energy profiles along with the hydrogen bond forming residues are tabulated in Table 5.

Table 5: Binding energies of galardin and its drug-like analog docked against the crystal structure of mPDF using accurate docking protocol of iGEMDOCK

\begin{tabular}{lllll}
\hline Sl. & Ligand & $\begin{array}{l}\text { Total } \\
\text { Energy }\end{array}$ & VDW & HBond \\
No. & & -127.186 & -107.735 & -19.4516 \\
\hline 1 & Galardin & 142.01 & -96.17 & -45.84 \\
\hline
\end{tabular}

From the Table 5, it is evident that the analog CID $5288446(142.01 \mathrm{kcal} / \mathrm{mol})$ shows a higher total binding energy (affinity) for mPDF protein than its corresponding parent compound galardin (127.186 $\mathrm{kcal} / \mathrm{mol}$ ). The van der Waals and hydrogen bond interaction energy of CID 5288446 was observed to be $-96.17 \mathrm{kcal} / \mathrm{mol}$ and $-45.84 \mathrm{kcal} / \mathrm{mol}$, respectively. The hydrogen bonding interaction between the analog CID 5288446 and mPDF protein was far better than its parent compound galardin. Therefore CID 5288446 can be considered as a better lead molecule since it interacts with MPDF with greater affinity and efficacy than its parent compound.

Interaction analysis of screened ligand and its parental compound with mPDF protein: The most important factor in protein-ligand interaction is the Van der Waals force of interaction and hydrogen bonding as they play an important role in determining the binding efficacy and orientation of drug-like molecule to its targets receptor or protein. The interaction profile of galardin and its derivative CID 5288446 with mPDF is tabulated in Table 6 .

Table 6: The Hydrogen bond and van der Waals Interacting Residues of mPDF protein with the putative mPDF inhibitor (CID 5288446) and its parental compound galardin

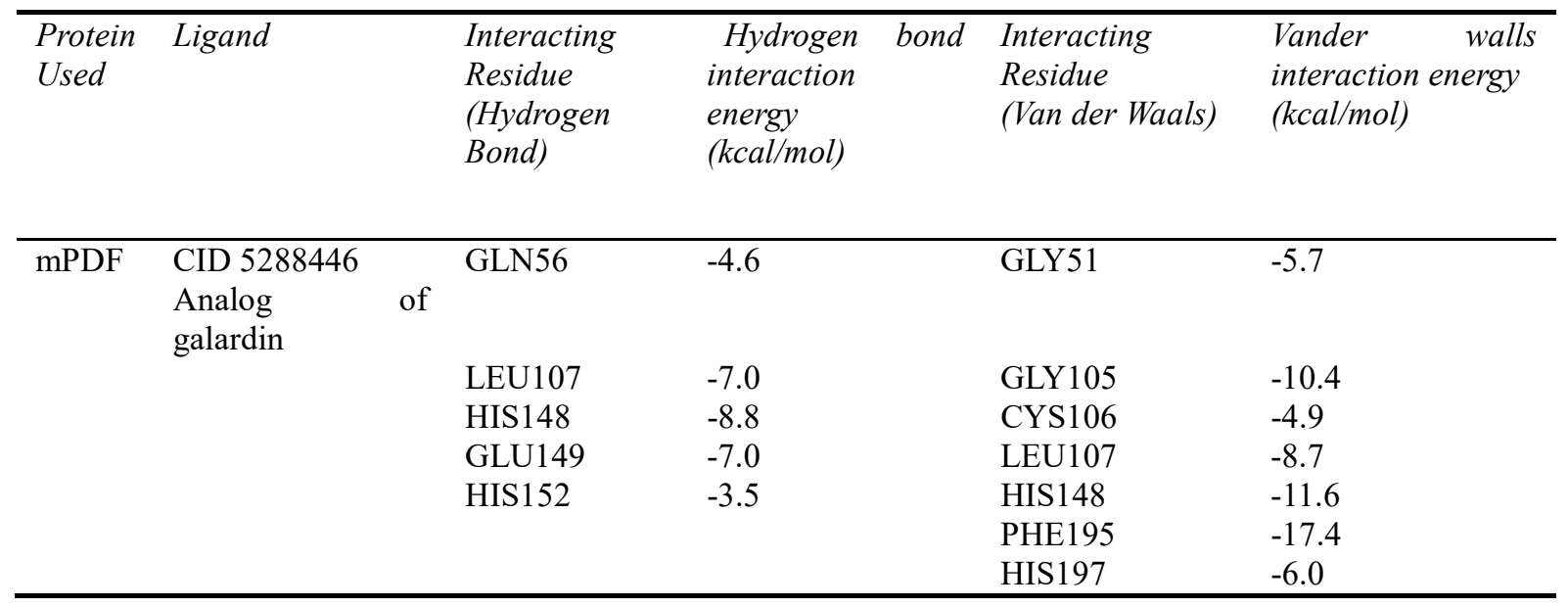

We observe from Table 5 that both hydrogen bonding and Van der Waals force of interaction profile of galardin as well as its derivative CID 5288446 are nearly similar. However, the efficacy of the interaction of the amino acids with the ligand in terms of energy is higher for CID 5288446 than its parental compound galardin. The hydrogen bond and Van der Waals force of interaction between the analog (CID 5288446) and $\mathrm{mPDF}$ protein is illustrated in Figure 1. 


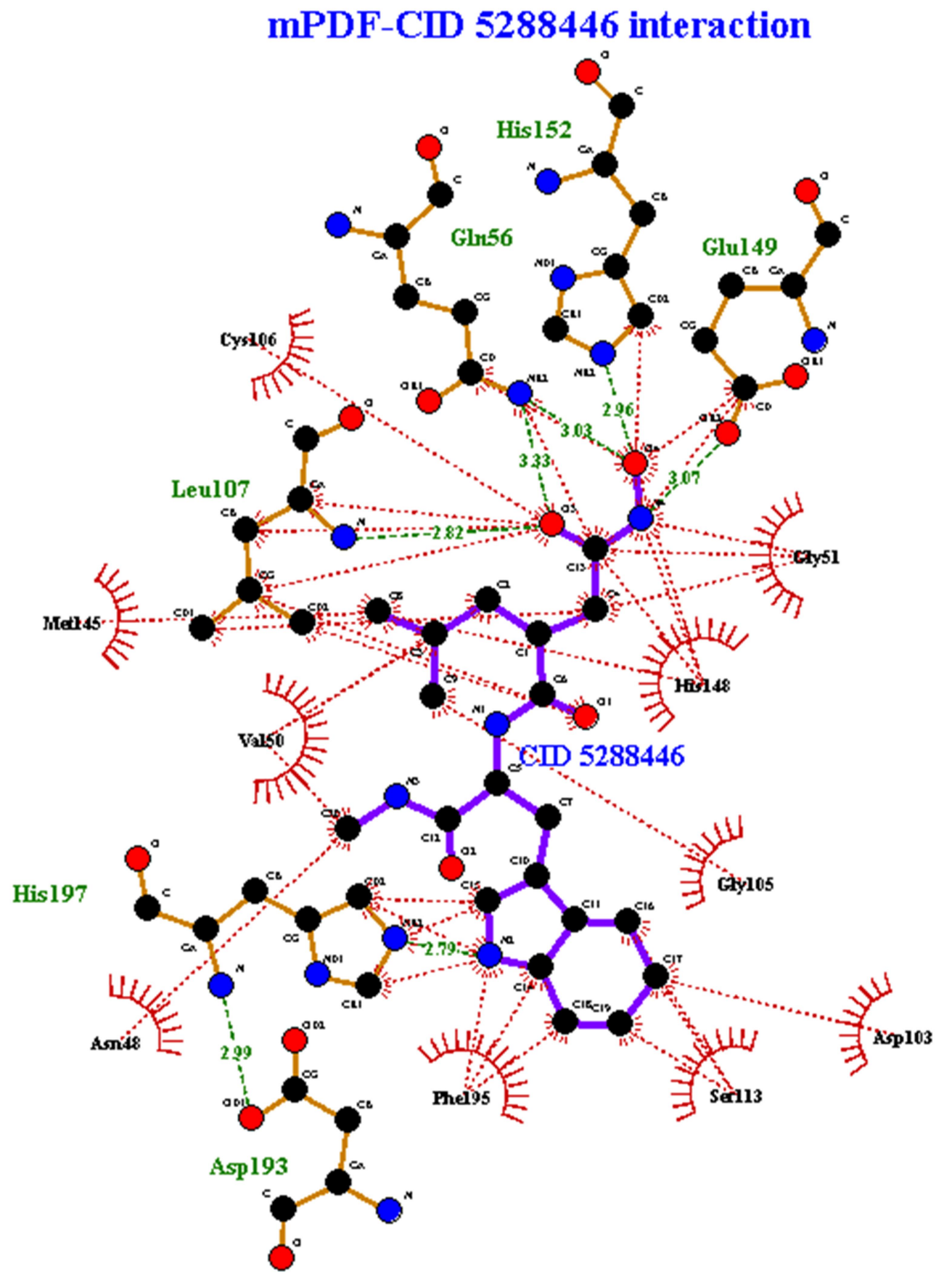

Figure 1: 2D representation of the hydrogen bonded and Van der Waals interactions of the putative inhibitor CID 5288446 (analog of galardin) with mPDF protein. The red dooted lines represent the Van der Waals force of interaction and the green colored dotted line represent the hydrogen bonded interaction between the ligand and protein.

As illustrated in Figure 1 the residues HIS152 and GLU56 of mPDF protein displayed strong hydrogen bonding with oxgen atom attached to hydroxyl group of the analog with corresponding bond length of $2.96 \AA$ and $3.03 \AA$. Additionally amino acids GLU56 and LEU107 of the target protein also forms a hydrogen bond 
with the carbonyl oxygen atom of the analog with a bond legth of 3.33 and $2.82 \AA$, respectively. GLU149 and HIS197 on the other hand forms a hydrogen bond with the nitrogen atom attached to the amine group of analog molecuele (CID 5288446) with a bond length of 3.07 and $2.79 \AA$, respectively. Results suggest that hydroxyl, carbonyl and amine groups of the analog act as the major functional groups assisting in the binding process of the analog (CID 5288446) with mPDF protein. The presence of CID 5288446 molecule interacting with residues lying close to the active site residues $\mathrm{H}^{132} \mathrm{EXXH}^{136}$ might hinder the activity of the protein and thereby inhibiting the function of mPDF in Mycobacterium tuberculosis. Therefore it can be suggested that the analog CID 5288446 of galardin as shown in Figure 2 can be a good lead molecule against mPDF protein based upon it efficiency in binding the ligand binding domain of mPDF protein with higher efficacy.

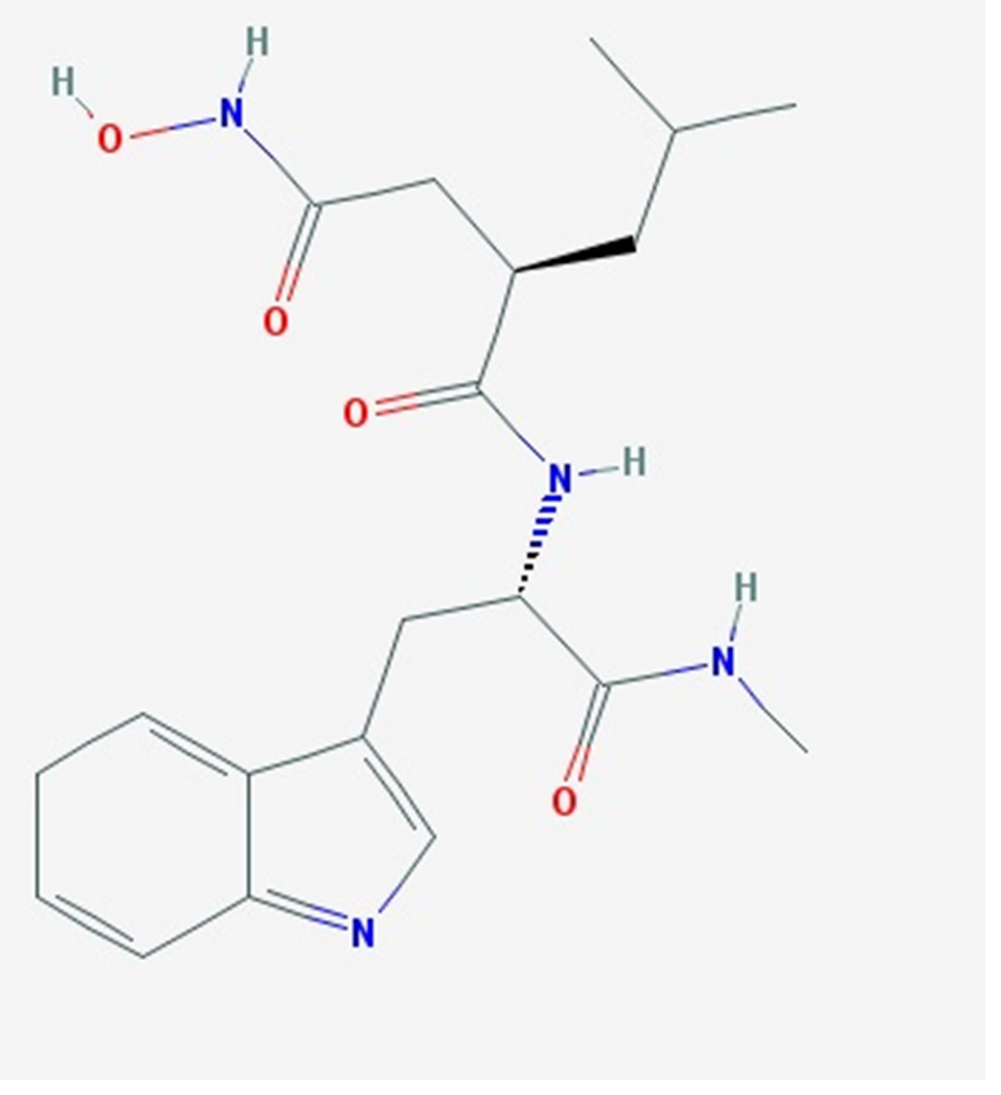

Figure 2: 2D structure of a putative mPDF inhibitor (CID 5288446)

Biological activity and toxicity studies of drug-like molecule and its parent compound: PASS (Prediction of Activity Spectra for Substances) software was used to predict the biological activity and toxic properties of the drug-like analog molecule (CID 5288446) as well as its parental compound (galardin). The prediction is based upon the structure-activity relationships calculated from the experimental data of known compound and then compared with data of the studied compound. The PASS algorithm also estimates the probability of the studied compound to be active or inactive as depicted by Pa (probability to be active) and Pi (probability to be inactive). The estimated biological activity and possible toxic effects for galardin and its derivative (CID 5288446) are tabulated in Table 7 and 8, respectively. 
Table 7: Prediction of the biological activity of Galardin and its derivative (CID 5288446) based on the structure-activity relationship function of PASS software

\begin{tabular}{lllll}
\hline Sl. No. & Ligand & $\boldsymbol{P a}$ & $\boldsymbol{P i}$ & Activity \\
\hline 1 & CID 5288446 & 0.698 & 0.003 & Metalloproteinase inhibitor \\
2 & Galardin & 0.716 & 0.002 & Metalloproteinase inhibitor \\
\hline
\end{tabular}

Pa: Probability of chemical compound to be active

Pi: Probability of the chemical compound to be inactive

Table 8: Prediction of toxic effects associated with galardin and it derivative (CID 5288446) based on the structure of structure-activity relationship function of PASS software.

\begin{tabular}{lllll}
\hline Sl. No. & Ligand & $\boldsymbol{P a}$ & $\boldsymbol{P i}$ & Toxic effects \\
\hline 1 & CID 5288446 & 0.387 & 0.135 & Edema \\
& (analog of Galardin) & 0.296 & 0.191 & Urinary retention \\
& & 0.269 & 0.248 & Occult bleeding \\
& & & \\
& & 0.770 & 0,050 & Edema \\
& Galardin & 0,766 & 0,055 & Urinary retention \\
& (Parent Compound) & 0,683 & 0,154 & Occult bleeding \\
& & 0,571 & 0,169 & Necrosis \\
& & 0,531 & 0,158 & Yawning \\
& & 0,672 & 0,239 & Retroperitoneal fibrosis \\
& & 0,633 & 0,229 & Visual acuity impairment \\
\hline
\end{tabular}

Pa: Probability of chemical compound to be active

Pi: Probability of the chemical compound to be inactive

We observe that both the analog (CID 5288446) and it parental structure (galardin) show similar metalloproteinase inhibitor activity and the probability for them to be an active inhibitor is also more of less same as depicted in Table 7. The predicted metalloproteinase inhibiting activity of CID 5288446 can be found in a US patent (US2010178259) where the compound CID 5288446 is used as a matrix metalloproteinase inhibitor to reduce fibrosis in skin (https://pubchem.ncbi.nlm.nih.gov/compound/5288446\#section=Top). Similarly, Galardin has been screened as an active compound as a metalloproteinase inhibitor in many bioassays namely AID-107338, 107348, 107358, 108007, 108308 and 108731 (https://pubchem.ncbi.nlm.nih.gov/compound/132519\#section=BioAssay-Results). As observed from Table 8 the toxic effects of the analog (CID 5288446) are less as compared to its parental compound (galardin). Additionally, for galardin PASS predicts few more toxic effects namely necrosis, yawning, retroperitoneal fibrosis and visual acuity impairment. The profiling of the possible adverse or toxic effects by PASS is based on clinical manifestations, detected in a handy or even in a single patient. Therefore, it can be proposed that the derivative (CID 5288446) of galardin can be used as a lead molecule to inhibit the mPDF of MTB with minimum toxicity and higher efficacy.

4. Conclusion: Based on results of oral bioavailability \& pharmacokinetics properties, affinity for mPDF protein and structure-activity relationship \& toxicity, it can be postulated that, (2R)-N'-hydroxy-N-[(2S)-3-(5H-indol-3-yl)-1-oxopropan-2-yl]-2-(2-methylpropyl) (CID 5288446) an analog of galardin can potentially prove to be better lead molecule than its parental compound in inhibiting the metalloproteinase activity of the mPDF protein in MTB. Therefore, identifying new therapeutic against MTB, which has been one of the prime motivating objectives considered while devising the study. Although the stability of the devised drug is yet to be tested computationally as well as impact in wetlab experimental studies.. The drug-like anlog CID 5288446 identified will aid in inhibiting activity of mPDF protein with minimum toxicity and higher efficacy. 


\section{REFERENCE}

[1] Daniel, T.M., Bates, J.H \& Downes, K.A. (1994). History of Tuberculosis. In: Bloom B.R., editor. Tuberculosis: Pathogenesis, Protection, and Control. American Society for Microbiology, 13-24, Washington, DC.

[2] World Health Organization (2016) http://www.who.int/tb/publications/factsheet_global.pdf?ua=1

[3] Jeanette, W.P.T., Pamela, T., David, B., Amelia, S.L., Yap, M.N., Xinyi, N., Jeyarj D, Sarah, L., Veronique, D., Mark, S., Samiul, H, Michael, C., Neil, S.R., Xia, Y., Beat, W., Kathryn, B., Thomas, D. \& Kakoli, M. (2006). Peptide Deformylase Inhibitors as Potent Antimycobacterial Agents. Antimicrobial Agents and Chemotherapy, 50(11), 3665-3673.

[4] Pichota, A., Duraiswamy, J., Yin, Z., Keller, T.H., Alam, J., Liung, S., Lee, G., Ding, M., Wang, G., Chan, W.L., Schreiber, M., Ma, I., Beer, D., Ngew, X., Mukherjee, K., Nanjundappa, M., Teo, J.W., Thayalan, P., Yap, A., Dick, T., Meng, W., Xu, M., Koehn, J., Pan, S.H., Clark, K., Xie, X., Shoen, C. \& Cynamon, M. (2008). Peptide deformylase inhibitors of Mycobacterium tuberculosis: synthesis, structural investigations, and biological results. Bioorganic \& Medicinal Chemistry Letters, 18, 6568-6572.

[5] Punia, P., Singh, S., Raj, T., Raguvaran, R., Chopra, M. \& Dahiya V. (2014). Docking Based Pharmacophore Model for Mycobacterium Tuberculosis Peptide Deformylase Inhibitors and Its Application in Drug Designing, International Journal of Pharmacy and Pharmaceutical Sciences, 6(2), 329-331.

[6] Adams, J.M. (1968). On the release of the formyl group from nascent protein. Journal of Molecular Biology, 33(3), 571-89.

[7] Livingston, D.M. \& Leder, P. (1969). Deformylation and protein biosynthesis. Biochemistry, 8(1), 435-43.

[8] Clements, J.M., Beckett, R.P., Brown, A., Catlin, G., Lobell, M., Palan, S., Thomas, W., Whittaker, M., Wood, S., Salama, S., Baker, P.J., Rodgers, H.F., Barynin, V., Rice, D.W. \& Hunter, M.G. (2001). Antibiotic Activity and Characterization of BB-3497, a Novel Peptide Deformylase Inhibitor. Antimicrobial Agents and Chemotherapy, 45(2), 563-570.

[9] Guilloteau, J.P., Mathieu, M., Giglione, C., Blanc, V., Dupuy, A., Chevrier, M., Gil, P., Famechon, A., Meinnel, T., \& Mikol, V. (2002). The Crystal Structures of Four Peptide Deformylases Bound to the Antibiotic Actinonin Reveal Two Distinct Types: A Platform for the Structure-based Design of Antibacterial Agents. Journal of Molecular Biology, 320(5), 951-62.

[10] Chikhi, A. \& Bensegueni, A. (2010). In Silico Study of the Selective Inhibition of Bacterial Peptide Deformylases by Several Drug. Journal of Proteomics \& Bioinformatics, 3, 061- 065.

[11] Fritsche, T.R., Moet, G.J. and Jones, R.N. (2004). Commercial broth microdilution panel validation and reproducibility trials for NVP PDF-713 (LBM 415), a novel inhibitor of bacterial peptide deformylase. Clinical Microbiology and Infection,10(9), 857-860.

[12] Lofland, D., Difuntorum, S., Waller, A., Clements, J.M., Weaver, M.K., Karlowsky, J.A., \& Johnson, K. (2004). In vitro antibacterial activity of the peptide deformylase inhibitor BB-83698. Journal of Antimicrobial Chemotherapy, 53, 664-668.

[13] Osborne, C.S., Neckermann, G., Fischer, E. and Pecanka, R., Yu, D., Manni, K., Goldovitz, J., Amaral, K., Dzink-Fox, J. \& Ryder, N.S. (2009). In Vivo Characterization of the Peptide Deformylase Inhibitor LBM415 in Murine Infection Models. Antimicrobial Agents and Chemotherapy, 53, 3777-3781.

[14] Yang, J.M. \& Chen, C.C. (2004). GEMDOCK: A generic evolutionary method for molecular docking. Proteins: Structure, Function, and Bioinformatics, 55, 288-304.

[15] Yang, J.M. (2004). Development and evaluation of a generic evolutionary method for protein-ligand docking. Journal of Computational Chemistry, 25, 843-857.

[16] Yang, J.M., Chen, Y.F., Shen, T.W., Kristal, B.S. \& Hsu, D. F. (2005). Consensus Scoring Criteria for Improving 5Enrichment in Virtual Screening. Journal of Chemical Information and Modeling, 45, 1134-1146.

[17] Laskowski, R. A., \& Swindells, M.B. (2011). LigPlot+: multiple ligand-protein interaction diagrams for drug discovery. Journal of Chemical Information and Modeling, 51, 2778-2786. 
[18] Borodina, Yu.V., Filimonov D.A., \& Poroikov, V.V. (1996). Computer-aided prediction of prodrug activity using the PASS system. Pharmaceutical Chemistry Journal, 30(12), 760-763.

[19] Filimonov, D.A., Poroikov, V.V., Karaicheva E.I. Kazarian R.K., Budunova A.P., Mikhailovskii E.M., Rudnitskikh A.V., Goncharenko, L.V., \& Burov, Yu.V. (1995). Computer-Aided Prediction of Biological Activity Spectra of Chemical Substances on the Basis of Their Structural Formulae: Computerized System PASS. Experimental and Clinical Pharmacology (Rus), 58 (2), 56-62.

[20] Lipinski, C.A. (2004). Lead- and drug-like compounds: the rule-of-five revolution. Drug Discovery Today: Technologies,1 (4), 337-341.

[21] Carpenter, T.S., Kirshner, D.A., Lau, E.Y., Wong, S.E., Nilmeier, J.P., \& Lightstone, F.C. (2014). A method to predict blood-brain barrier permeability of drug-like compounds using molecular dynamics simulations. Biophysics Journal. 107(3), 630-641.

[22] Sigel, A., \& Sigel, H. (2007). The Ubiquitous Roles of Cytochrome P450 Proteins: Metal Ions in Life Sciences. Vol. 3. John Wiley \& Sons, Ltd.

[23] Amin, L. (2013) 'P-glycoprotein Inhibition for Optimal Drug Delivery', Drug Target Insights., Vol.7, pp.27-34. 